\title{
Collectif, Rappresentazioni della natura nel Medioevo
}

\section{Antonella Sciancalepore}

\section{OpenEdition}

\section{Journals}

Édition électronique

URL : https://journals.openedition.org/ccm/8125

DOI : $10.4000 / \mathrm{ccm} .8125$

ISSN : 2119-1026

\section{Éditeur}

Centre d'études supérieures de civilisation médiévale/Université de Poitiers

\section{Édition imprimée}

Date de publication : 1 septembre 2021

Pagination : 281-283

ISBN : 978-2-490783-10-6

ISSN : 0007-9731

Référence électronique

Antonella Sciancalepore, "Collectif, Rappresentazioni della natura nel Medioevo », Cahiers de civilisation médiévale [En ligne], 255 | 2021, mis en ligne le 01 septembre 2021, consulté le 08 février 2022. URL : http://journals.openedition.org/ccm/8125; DOI : https://doi.org/10.4000/ccm.8125

La revue Cahiers de civilisation médiévale est mise à disposition selon les termes de la Licence Creative Commons Attribution - Pas d'Utilisation Commerciale - Pas de Modification 4.0 International. 
Rappresentazioni della natura nel Medioevo, Giovanni CATAPANo et Onorato Grassi (dir.), Florence, Sismel (Micrologus'Library, 94), 2019.

Le présent volume réunit les actes du congrès de la Société italienne pour l'étude de la philosophie médiévale, qui a eu lieu à Padoue du 24 au 27 mai 2017. Le rôle dynamique du milieu padouan dans la réalisation du congrès et des actes est évident : la moitié des contributeurs sont affiliés à l'université de Padoue. Après l'introduction, les 18 articles - chacun suivi d'un résumé en anglais - se succèdent sans être répartis en sections; un article de conclusion, $24 \mathrm{p}$. d'illustrations en couleurs et les index (auteurs et ouvrages, manuscrits cités) terminent le volume.
Dans leur préface (p. VII-XIV), Giovanni Catapano et Onorato Grassi fournissent un utile bilan des études sur la nature au Moyen Âge. Les éditeurs mettent en évidence la richesse des définitions données au terme «nature », et les évolutions que ce terme a subi à la suite des mutations scientifiques, philosophiques et plus généralement culturelles tout au long de la période médiévale. En effet, les textes qui composent le volume décrivent une nébuleuse très variée d'apports originaux au sujet de la nature et de ses représentations. La période envisagée $-\mathrm{du}_{\mathrm{IV}}^{\mathrm{e}} \mathrm{au}$ $\mathrm{XV}^{\mathrm{e}} \mathrm{s}$. - et la variété des savoirs médiévaux abordés - la philosophie, la mathématique, la physique, 
la pharmacopée, l'alchimie, le droit - et des formes artistiques traitées - la poésie lyrique, la musique, l'art monumental, l'enluminure, l'architecture - font de ce volume un des rares exemples de pluridisciplinarité authentique. De plus, à l'image de la polysémie médiévale de «nature », les auteurs des articles aussi convoquent des interprétations différentes de ce terme et de ce qui constitue sa représentation.

En conclusion du volume, le fondateur et éditeur de la Micrologus'Library, Agostino Paravicini Bagliani, souligne aussi la définition très souple du thème tout au long des articles. Il isole deux catégories dans lesquelles ranger les articles : les uns qui s'occupent des représentations de la nature, et du rapport entre nature et réalité sensible, et les autres qui traitent des problèmes relatifs à l'histoire de la pensée médiévale. Je proposerais de décrire les approches de ce volume à travers une autre image. Osant emprunter un modèle topologique-conceptuel médiéval, je dirais que les articles en question se répartissent en trois cercles concentriques, en fonction de leur focalisation et de leur interprétation du thème du volume.

Dans le cercle intérieur, nous pourrions localiser les articles qui s'occupent de retracer et explorer les définitions médiévales de «natura», et de leurs transformations au fil du temps, qui ont des retombées à la fois lexicales, philosophiques, physiques, anthropologiques et juridiques. L'exploration commence au Moyen Âge avec Saint Augustin, dont Enrico Moro analyse le lexique employé pour décrire le monde issu de la création; d'après le commentaire de l'évêque d'Hippone sur le livre de la Genèse, la nature est le « potentiel causal » inscrit dans les êtres vivants au moment de la création (p. 27-40). À partir du XII ${ }^{\mathrm{e}} \mathrm{s}$., avec l'arrivée en Occident des traductions d'Aristote, la discussion sur le concept de nature devient aussi l'occasion d'une translation entre le savoir philosophique du haut Moyen Âge, les écrits d'Aristote nouvellement découverts et ses commentaires arabes et occidentaux. C'est le cas de Thomas d'Aquin qui, dans ses commentaires à la Métaphysique, harmonise la définition de «natura » de Boétie avec celle d'Aristote, avant d'en donner une nouvelle interprétation, incluant aussi l'idée d'après laquelle la nature comprendrait aussi des substances artificielles (Fabrizio Amerini, p. 97-110). La distinction entre naturel et artificiel sera abordée plus explicitement par l'école de physique parisienne au XIV $\mathrm{s}$. : Nicole Oresme, Albert de Saxe, Jean Buridan et
Marsile d'Inghen apportent chacun une réponse différente à cette question, mais admettent également une continuité entre nature et art, où l'art est un principe accidentel, et non principal, des choses artificielles (Fabio Zanin, p. 193-206).

De la définition philosophique de «natura » procède la connaissance des causes qui règlent la réalité et de leurs possibles modifications. $\mathrm{Au} \mathrm{x} \mathrm{x}^{\mathrm{e}} \mathrm{s}$, dans son commentaire au Calculus de Victoire d'Aquitaine $\left(v^{e} s.\right)$, Abbon de Fleury affirme que les causes de l'univers, qui sont aussi les causes intrinsèques de l'essence divine, sont mathématiques, comme le démontre, d'après lui, la commensurabilité des phénomènes physiques (Clelia V. CRIALESI, p. 41-58). Une approche différente de la nature est celle de l'alchimie : Paola Carusi nous ramène dans le débat sur la définition aristotélicienne de nature qui oppose Avicenne et Al-Tugrāâ'ī; l'interprétation du dernier de la mixis d'Aristote comme une admission de la possibilité de la transmutation semblerait avoir eu un impact qui va au-delà de l'alchimie arabe, pour arriver jusqu'à Thomas d'Aquin (p. 59-81).

La définition de «natura » est particulièrement cruciale aussi pour connaître et diriger l'homme. Andrea Porcarelli s'interroge sur cet aspect anthropologique chez Thomas d'Aquin : d'après son Liber de causis, l'être humain serait caractérisé par sa capacité d'être éduqué - qualité unique qui procéderait de la place de l'homme, qui se situe à la frontière entre devenir et éternité, entre corps et esprit (p. 111-124). Si cette définition a une grande portée dans la théorie pédagogique moderne, la nature a déjà un rôle crucial dans la jurisprudence du Moyen Âge. $\mathrm{Au} \mathrm{XIII}^{\mathrm{e}}$ s., Jean de la Rochelle, dans un traité qui servira d'inspiration pour la section juridique de la Summa d'Alexandre de Hales, fait un bilan des significations de nature et de son rapport avec l'action humaine et le libre arbitre, pour définir le droit naturel comme l'«impressio » de la loi éternelle dans les créatures rationnelles (Riccardo Saccenti, p. 125-137). La définition de droit naturel et l'utilisation du concept philosophique de nature sont sujettes au changement dans la jurisprudence médiévale : Giovanni Rossi nous montre le passage, au XIV s., depuis l'utilisation de la nature comme la garantie du travail du législateur - dans le Dictionarium iuris d'Alberico da Rosate - à une considération plus approfondie de la nature, en tant que cause des mouvements et des pulsions humaines - dans 
les commentaires de Baldo degli Ubaldi au Corpus Iuris Civilis (p. 139-153). Iolanda Ventura évoque une autre facette du rapport entre connaissance de la nature et homme, la pharmacologie : la diffusion de la Physica d'Aristote dans les milieux académiques détermine un changement dans les curricula universitaires, vers un renouvellement du rapport entre étude de la nature du monde et étude de la physiologie humaine (p. 259-274).

Dans le deuxième cercle, je localiserai les articles qui sont encore bien centrés sur le sujet de la représentation de la nature, mais qui interprètent cette nature surtout dans le sens d'espace naturel, composé d'animaux et de plantes, et de sa représentation littéraire et artistique. Alessandro Scafi ouvre le volume en parlant d'une nature paradoxale, celle du paradis terrestre, qui est à la fois parfaite et sensible, à travers les textes de Thomas d'Aquin, qui décrit l'utopie d'un homme libre des passions et maîtrisant la nature dans le jardin d'Éden (p. 3-25). La littérature médiévale regorge d'images utopiques de la nature. D'après Valeria Russo, la lyrique d'amour provençale utiliserait les images naturelles - et les oiseaux en particulier - comme expression des aspects les plus spirituels du « Je » poétique, bien qu'avec des effets différents (p. 83-96). L'évocation de la nature à fins expressives est aussi le sujet de l'article de Paola Dessì, qui d'ailleurs se focalise sur l'utilisation des images sidérales et florales dans la construction de l'identité de la dame dans les madrigaux de Bartolino de Padoue (p. 155-172).

Deux articles examinent également la représentation visuelle de la nature physique. Chiara Ponchia et Federica Toniolo proposent un aperçu des changements dans l'iconographie des animaux et des plantes dans les manuscrits médiévaux, qui conduisent la nature des marges au centre de la page (p. 241-258). La nature est au cœur d'une tension entre surface, cadre et centre de la représentation aussi dans l'art roman d'après l'étude de Xavier Barral i Altet : la terre, l'eau et les animaux qui l'habitent recréent les frontières du cosmos à l'intérieur de l'espace architectonique (p. 275-290). La nature reste un modèle de l'organisation de l'espace aussi au seuil de la Renaissance, à un niveau plus théorique, cependant : Leon Battista Alberti considère la nature physique comme un ensemble de lois qui peuvent être appliquées à l'architecture, et le corps humain comme un modèle des proportions à utiliser dans l'édification (Remy Simonetti, p. 291-303).

Enfin, dans le troisième cercle, le plus extérieur, nous pourrions placer les études qui interprètent le thème du volume dans son acception la plus large et flexible: ce sont les articles qui traitent de « la nature de X », c'est-à-dire des caractéristiques qui définissent l'existence d'un élément, d'un phénomène ou d'une discipline dans la culture médiévale. Bien que leur rapport avec le thème du volume paraisse quelquefois éloigné, ils constituent des articles riches d'intérêt scientifique. Antonio Lovato s'interroge sur la nature de la musique chantée chez le Lucidarium de Marchettus de Padoue, et observe la nouvelle attention, au XIII ${ }^{\mathrm{e}}$ s., portée aux potentialités communicatives du son de la voix, capable de transmettre des messages non verbaux de façon parallèle et complémentaire à la peinture (p. 173-192). Chiara Beneduce explore la nature du toucher et les évolutions que la définition du toucher subit aux XIV et XV $\mathrm{XV}^{\mathrm{e}} \mathrm{s}$.; Jean Buridan, en particulier, se fait l'interprète d'un nouvel intérêt à la question des sens, qui combine une relecture personnelle d'Aristote avec des éléments de la tradition médicale (p. 207-220). La partie des sens et de la perception est au centre aussi de l'article de Zuleika Murat, qui passe en revue les modalités d'ostension des reliques saintes à Venise aux XIII et XIV ${ }^{\mathrm{e}} \mathrm{s}$.; la stimulation de l'odorat, du toucher et de la vue est garantie par les caisses dans lesquelles les corps saints sont rendus accessibles aux pèlerins (p. 221-239).

En général, le volume ne trahit pas les attentes du lecteur en quête d'un large éventail de pistes d'exploration sur la nature au Moyen Âge, dans toutes ses significations. La variété risque de nuire à la cohérence générale du volume, mais elle n'empêche pas que chaque article soit en dialogue avec au moins un autre article de la collection, grâce aux concordances des champs explorés et à l'influence des auctoritates pour la connaissance de la nature au Moyen Âge, comme Aristote et Thomas d'Aquin.

Antonella SCIANCALEPORE

Université catholique de Louvain - Institut de civilisations, arts et lettres/Centre d'études sur le Moyen Âge et la Renaissance 